\title{
ARTICLE OPEN Multi-loop node line states in ternary MgSrSi-type crystals
}

\author{
Jinling Lian ${ }^{1}$, Lixian $\mathrm{Yu}^{1}$, Qi-Feng Liang ${ }^{1}$, Jian Zhou $\mathbb{D}^{2}$, Rui $\mathrm{Yu}^{3}$ and Hongming Weng $\mathbb{D}^{4,5}$
}

Node line band-touchings protected by mirror symmetry (named as $m$-NLs), the product of inversion and time reversal symmetry $\mathrm{S}=\mathrm{PT}$ (named as s-NLs), or nonsymmorphic symmetry are nontrivial topological objects of topological semimetals in the Brillouin Zone. In this work, we screened a family of MgSrSi-type crystals using first principles calculations, and discovered that more than 70 members are node-line semimetals. A new type of multi-loop structure was found in AsRhTi that a s-NL touches robustly with a $m$-NL at some "nexus point", and in the meanwhile a second $m$-NL crosses with the $s$-NL to form a Hopf-link. Unlike the previously proposed Hopf-link formed by two $s$-NLs or two $m$-NLs, a Hopf-link formed by a $s$-NL and a $m$-NL requires a minimal three-band model to characterize its essential electronic structure. The associated topological surface states on different surfaces of AsRhTi crystal were also obtained. Even more complicated and exotic multi-loop structure of NLs were predicted in AsFeNb and PNiNb. Our work may shed light on search for exotic multi-loop node-line semimetals in real materials.

npj Computational Materials (2019)5:10; https://doi.org/10.1038/s41524-018-0147-y

\section{INTRODUCTION}

The band crossings of the conduction and valence bands in a topological semimetal are interesting topological objects of Brillouin Zone (BZ) which bring about unique electronic structures and electrical properties, such as giant magnetoresistance, parity anomaly and "drum-head" states at material's surfaces. ${ }^{1,2}$ Depending on the dimensionality of band crossings, topological semimetals are classified into three categories, the Weyl semimetals $(\mathrm{WSMs})^{3-8}$ or Dirac semimetals (DSMs), ${ }^{9-11}$ node-line semimetals (NLSMs), ${ }^{12-41}$ and node-surface semimetals (NSSMs). ${ }^{42,43}$ Unlike the DSMs and WSMs whose band crossings take place at discrete points in the $B Z$, the band crossings of NLSMs form closed loops. When circling around these loops, an electron picks up an nontrivial Berry phase $\pi$ in its wave function, whose effect can be detected by transporting measurements. Though having been extensively proposed in graphene networks, $^{13}$ anti-perovskites, ${ }^{14,15} \mathrm{SrlrO}_{3}{ }^{20} \mathrm{TlTaS}_{2}{ }^{17} \mathrm{BaTaS}^{42} \mathrm{HfC}^{29}$ $\mathrm{CaP}_{3} / \mathrm{CaAs}_{3}{ }^{30,31}$, and $\mathrm{Co}_{2} \mathrm{MnGa}^{35}$ etc, the direct evidence of existence of node-line (NL) states in real materials is rare. ${ }^{18,25-27}$ Finding new materials with clean and robust NL band crossing around the fermi level is still a demanding task in the field of condensed matter physics.

Three types of NLs have been discovered based on their protecting symmetry. ${ }^{2}$ The first type of NLs is protected by mirror symmetry, which is named as $m-\mathrm{NL}$ in this work and shown schematically in Fig. 1a. Due to the mirror symmetry, the $m-\mathrm{NL}$ is pinned to the invariant plane of the mirror symmetry. The second type of NLs is protected by the combination of time-reversal symmetry $T$ and inversion symmetry $P$, i.e., $\mathrm{S}=\mathrm{PT}$. This type of NLs, named as s-NL here, can present at any region of the BZ as shown at the right of Fig. 1a. The last type of NLs is protected by nonsymmorphic symmetries and usually appears at the boundary of $B Z .{ }^{20,42}$
Recently, there rises a new trend of investigating NLSMs with multiple NL loops. ${ }^{28,29,33-36,38}$ In those NLSMs, NL loops may intersect with each other and entangle into a variety of structures, such as node-net, ${ }^{38}$ node-chain ${ }^{28}$, and Hopf-link, ${ }^{32-36}$ etc. For example, two $m$-NLs will be stuck together at some points dubbed as "nexus points" on the cross-line of two invariant planes of mirror symmetries (see in Fig. $1 \mathrm{~b}$ ). ${ }^{44}$ In the case of two s-NLs, the $s$-NLs can be separated, touched or crossed with unrestricted locations in the BZ (see in Fig. 1c). The crossed s-NLs are also called Hopf-link due to their topological invariant being the Hopf-link number. ${ }^{33}$ While the existence of multiple-loop NLs has been realized in photonic lattice, ${ }^{45}$ their existence in fermionic systems has not been identified thus far.

In this work, using first principles calculations, we screened the family of MgSrSi-type crystals which consists of 660 members, and found more than 70 compounds are NLSMs showing a variety of NL structures. The NLs are protected by the mirror symmetry or the $S$ symmetry contained in the Pnma space group of MgSrSitype crystals. Importantly, in contrast to a previous report in which the member AsRhTi was predicted to be a NLSM with a single NL band crossing, 22 we found in this material a new type of multiloop NL structure as shown in Fig. $1 \mathrm{~d}$, where a $s$-NL sticks to a $m$ $\mathrm{NL}$ at some "nexus point" (denoted by $O$ ) and penetrates the invariant plane of $m-\mathrm{NL}$ at some general point (denoted by $E$ in Fig. (1d)). Interestingly, we also found a third $m$-NL crosses the $s-\mathrm{NL}$ and a Hopf link is formed. Unlike the case of two crossed $m$ NLs or two crossed s-NLs, this novel multi-loop NL structure requires a minimal three-band model to describe its essential electronic structure. Even more exotic multi-loop NL structures were further uncovered in AsFeNb and PNiNb. Some of the NLSMs show very clean band structures at the fermi level without other trivial bands. Our work therefore provides a promising platform for the material realization of new topological semimetals with exotic NL structures.

\footnotetext{
${ }^{1}$ Department of Physics, Shaoxing University, 312000 Shaoxing, China; ${ }^{2}$ National Laboratory of Solid State Microstructures and Department of Materials Science and Engineering, Nanjing University, 210093 Nanjing, China; ${ }^{3}$ School of Physics and Technology, Wuhan University, 430072 Wuhan, China; ${ }^{4}$ Beijing National Laboratory for Condensed Matter Physics, and Institute of Physics, Chinese Academy of Sciences, 100190 Beijing, China and ${ }^{5}$ Songshan Lake Materials Laboratory, Guangdong 523808, China Correspondence: Q-F. Liang (qfliang@usx.edu.cn) or Rui Yu (ruiyu@whu.edu.cn)
}

Received: 9 August 2018 Accepted: 11 December 2018

Published online: 21 January 2019 
(a)

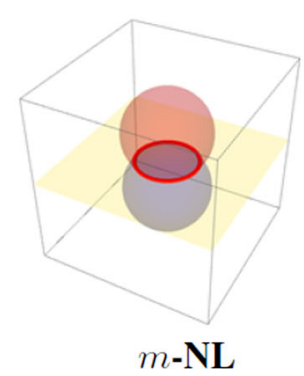

(c)

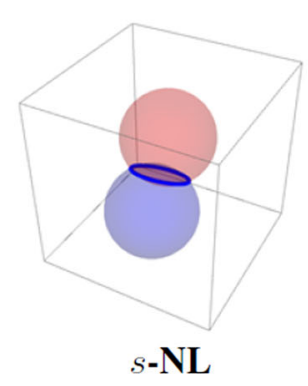

$s-\mathrm{NL}$ (b)

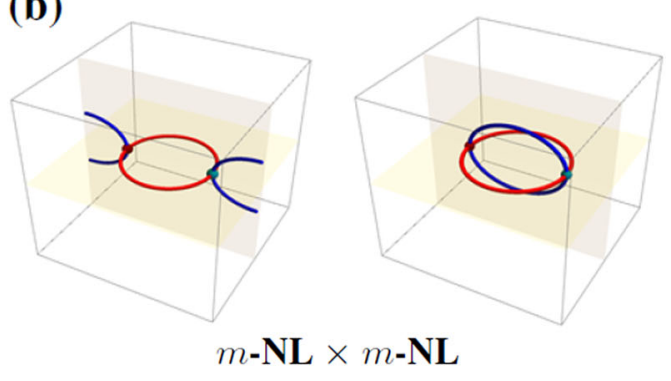

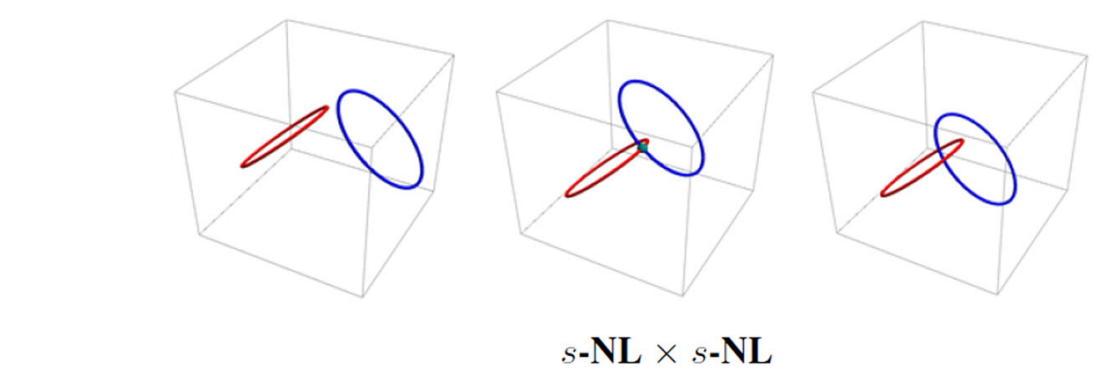

(d)

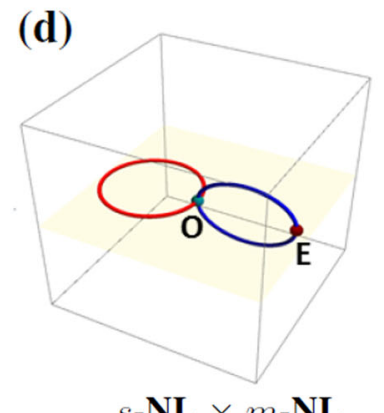

Fig. 1 Possible NL structures in BZ. a The $m$-NL protected by mirror symmetry (left) and the $s$-NL protected by symmetry $S=P T$ (right). b A pair of touched $m$-NLs associated with two vertical mirror planes. The small spheres located at the touching points of two NLs are also dubbed as "nexus points". c A pair of separated, touched and crossed s-NLs depending on their distance in the BZ. d A pair of touched $s$-NL and $m$-NL. Except the nexus point $\mathrm{O}$, the $s$-NL also penetrates the invariant plane of the $m$-NL through another isolated point marked as $\mathrm{E}$

\section{RESULTS AND DISCUSSION}

\section{Multi-loop NLs in AsRhTi}

The group of MgSrSi-type crystal is consisted of 660 members as documented in ICSD. ${ }^{46-56}$ It takes a Pnma space group which contains a mirror plane $m_{y}$, two glide mirror planes $\tilde{m}_{x}$ and $\tilde{m}_{z}$ and the space inversion $P$. On the $k_{x}=0$ and $k_{z}=0$ invariant planes, the glide planes $\tilde{m}_{x}$ and $\tilde{m}_{z}$ act in the same way as the normal mirror planes, ${ }^{20}$ while on the boundary of $B Z$, they should be treated differently since their fractional translations may lead to additional band degeneracy. If the time reversal $T$ is also a symmetry, the compounds become symmetrical under the composed operation $\mathrm{S}=\mathrm{PT}$. Both conditions of existence of the $s$-NL and $m$-NLs are therefore fulfilled in MgSrSi-type crystals. In Fig. (2a) we plotted the crystal structure of a prototype compound, AsRhTi and the two key symmetries for NLs, a mirror plane $m_{y}$ and inversion symmetry $P$ are highlighted. It can be found that in the unit cell of AsRhTi it contains two layers of atoms and the plane of the atom layers is overlapped with the mirror plane of $m_{y}$. The inversion center, on the other hand, is off the atom layers and locates at the middle of two neighboring layers. The corresponding Brillouin Zone and high-symmetry paths are also shown below the crystal structure of AsRhTi.

Let us show here that the valence and conduction bands of AsRhTi do cross and produce a multi-loop NL structure. We plotted the GGA band structure of AsRhTi in Fig. $2 \mathrm{~b}$ where one can readily find that the valence and conduction bands cross at the intermediate points of $\Gamma-X$ and $\Gamma-Z$, indicating a NL lying in the invariant plane $k_{y}=0$ of the mirror symmetry $m_{y}$. In order to demonstrate the NL structure more clear, we further plotted in Fig. $2 \mathrm{c}$ the $2 \mathrm{D}$ band structure of AsRhTi on the $k_{y}=0$ plane with varying $k_{x}$ and $k_{z}$. Since the band structure is symmetrical under the transformations $k_{x} \rightarrow-k_{x}$ and $k_{z} \rightarrow-k_{z}$, only the region of $k_{x}>$ 0 and $k_{z}>0$ is used for simplicity. From the profile of the energy difference of three bands that has been projected on the bottom of Fig. $2 c$, one sees a central NL $a$ surrounding the $\Gamma$ point. Outside the NL $a$ is a $m-N L \beta$. Outmost is a third $m-N L ~ \gamma$ that encloses the $U$ point. It is the $m_{y}$ provides the needed protection for the three
$m$-NLs. Interestingly, besides these in-plane $m$-NLs, we also find an isolate band-touching point $\mathrm{E}$ outside the NL $\gamma$. This bandtouching point $E$ is more obviously seen in the $2 \mathrm{D}$ energy band structure where it is highlighted by a dotted circle in Fig. 2c.

The detail of the NLs near the point $E$ is revealed by a 3D profile of energy differences of bands in Fig. 2d, where a denser discretion of $B Z$ is adopted to obtain the energy bands with the tight-binding hamiltonian constructed by the MLWF method. In this band-crossing profile, an extra NL $\delta$ vertical to $k_{y}=0$ plane is discovered. Since NL $\delta$ does not lie in any high symmetry path or plane, it must be an s-NL that is protected by symmetry $\mathrm{S}$. More detailly, the $s-\mathrm{NL} \delta$ is found to stick to the $m-\mathrm{NL} \beta$ on a nexus point and the point $\mathrm{E}$ is the very point that $\mathrm{NL} \delta$ penetrates through the $k_{y}=0$ plane. Another interesting feature in Fig. $2 \mathrm{~d}$ is that the $m-\mathrm{NL}$ $\gamma$ crosses the $s$-NL and the two form a Hopf-link. Previously Hopflinks made of two $m$-NLs or two $s$-NLs have been already proposed and it is argued that for the first one needs a minimal four-band effective model to describe the electronic structure of the Hopflink, ${ }^{35}$ while for the later one only needs a minimal two-band model. ${ }^{33,34}$ Here we demonstrate that a Hopf-link can be made of a $s-\mathrm{NL}$ and a $\mathrm{m}-\mathrm{NL}$, and its corresponding electronic structure is correctly described by a minimal three-band model given below.

The reason that why we need a minimal three-band model to describe the multi-loop NL structure of Fig. $1 d$ and of Fig. $2 d$ is obvious: The $m-\mathrm{NL}$ is only produced by a pair of bands with opposite mirror parities. A robust and isolate band crossing point $E$ on the invariant plane $k_{y}=0$, however, is only possible when the crossing bands have equal mirror parities. Otherwise there would be a $m$-NL passing through the isolate point. ${ }^{29}$ The general form of the three-band hamiltonian should be written as,

$$
H(k)=\left[\begin{array}{ccc}
H_{11}(k) & H_{12}(k) & H_{13}(k) \\
& H_{22}(k) & H_{23}(k) \\
\dagger & & H_{33}(k)
\end{array}\right] .
$$

Since the system preserves the symmetry $S$, the imaginary part of the off-diagonal element $H_{n m}(k)(n, m=1,2,3$, and $n \neq m)$ vanishes. On the other hand, The mirror symmetries, i.e., $\tilde{m}_{x}, m_{y}$, 
(a)

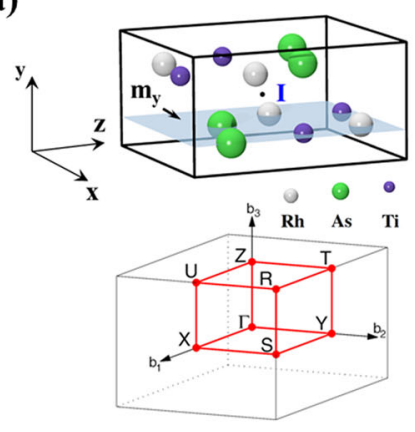

(c)

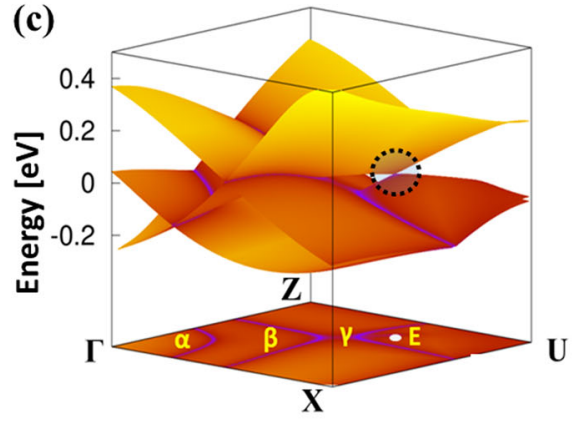

(b)

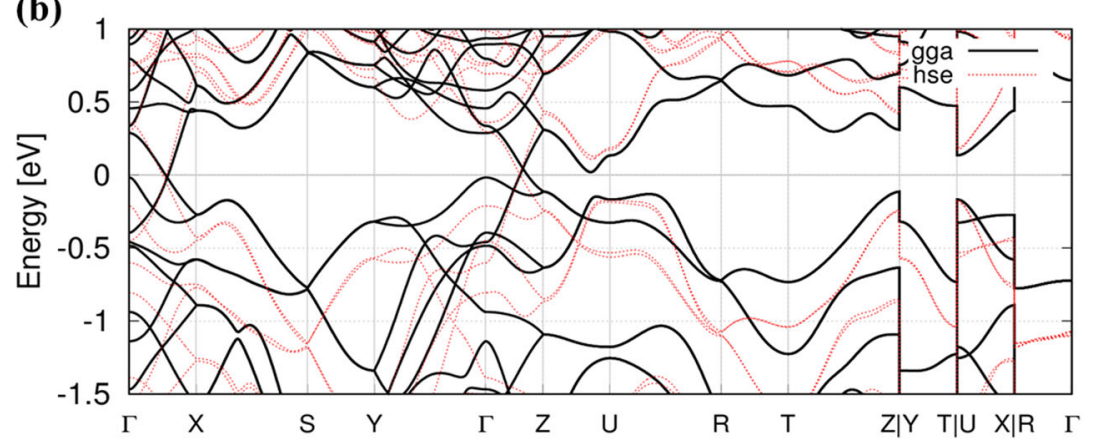

(d)

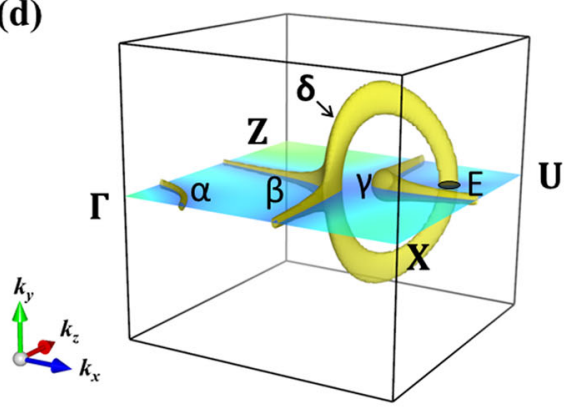

(e)

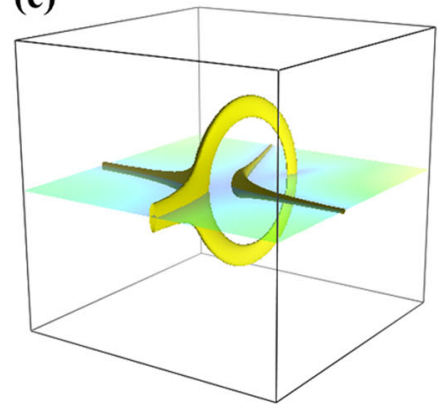

Fig. 2 NLs band crossings of AsRhTi. a The crystal structure and Brillouin Zone of AsRhTi. The mirror plane of $m_{y}$ and space inversion symmetry $P$ are highlighted. $\mathbf{b}$ Band structure of AsRhTi. Both band structures obtained from the GGA and HSE06 calculations are presented by solid and dotted lines, respectively. c Band structure on the invariant plane $k_{y}=0$. Only three bands at the fermi level are plotted and the energy differences between these bands are projected on to the bottom of the figure. Three $m$-NLs $a, \beta$, and $\gamma$ are highlighted. The point $\mathrm{E}$ denoted an isolated band crossing point on the $k_{y}=0$ plane. $\mathbf{d}$ The band crossings in the 3D BZ. The point E should be an isolation intersection point of the $s-\mathrm{NL} \delta$ and the $k_{y}=0$ plane which is further highlighted by the dotted circle. e The band crossing calculated from the $k p$ model in Eq. (2). The dimensionless parameters are set as $\varepsilon_{1}=-0.346, \varepsilon_{2}=-0.865, \varepsilon_{3}=-0.26, a_{1}=1.0, a_{2}=1.0, a_{3}=0.15, b_{1}=0.05, b_{2}=$ $1.0, b_{3}=0.15, c_{1}=1.0, c_{2}=0.05, c_{3}=0.15, \lambda_{1}=0.588$ and $\lambda_{2}=0.588$

and $\tilde{m}_{z}$, lay on the entries another constraint that the diagonal element $H_{n n}(k)$ should be an even function of $k_{x}, k_{y}$, and $k_{z}{ }^{15}$ For the off-diagonal entry $H_{n m}(k)$ with $n \neq m$, it becomes an even (odd) function of $k_{i}(i=x, y, z)$ if the orbital $n$ and $m$ have the equal (opposite) mirror parities with respect to symmetry $m_{i}{ }^{15}$ The above symmetry consideration helps us to reduce the hamiltonian of Eq. (1) to a simpler form up to a second order of $k$,

$$
H(k)=\left[\begin{array}{ccc}
0 & \lambda_{1} k_{y} & \lambda_{2} k_{y} \\
& \varepsilon_{1}+a_{1} k_{x}^{2}+b_{1} k_{y}^{2}+c_{1} k_{z}^{2} & \varepsilon_{3}+a_{3} k_{x}^{2}+b_{3} k_{y}^{2}+c_{3} k_{z}^{2} \\
\dagger & & \varepsilon_{2}+a_{2} k_{x}^{2}+b_{2} k_{y}^{2}+c_{2} k_{z}^{2}
\end{array}\right] .
$$

Here we supposed that the second and third orbitals have the equal mirror parities opposite to that of the first. A constant term $H_{11} \hat{l}$ has been subtracted from the original Hamiltonian because of its irrelevance to the structure of NLs. The parameters of Eq. (2) are chosen dimensionless for simplicity. By choosing suitable values of parameters $\varepsilon_{i}, a_{i}, b_{i}, c_{i}$, and $\lambda_{j}(i=1,2,3$ and $j=1,2)$, the main features of multi-loop NL structure of Fig. $2 \mathrm{~d}$ are well reproduced as shown in Fig. 2e.

The nontrivial electronic structure of a NLSM is revealed by its topological surface states (SSs). In a slab, the projection of a NL in the bulk BZ onto the $2 \mathrm{D} B Z$ will divide it into regions of different topological orders characterized by $Z_{2}$ topological charge $v_{1}^{57}$

$\nu=\frac{1}{\pi} \int_{-\pi}^{\pi} d k_{\perp} \sum_{n \in \text { occ. }}\left\langle n, k\left|i \partial_{\mathrm{k}_{\perp}}\right| n, k\right\rangle \bmod 2$,

where $|n, k\rangle$ denotes the Bloch eigenstate and $k_{\perp}$ is the component of momentum normal to the slab. In the regions of $v=1$, there exists in-gap topological surface states at each $k$ point, forming the so called 2D "drum-head" states. ${ }^{57}$ In Fig. 3a, b we have shown the surface band structures of AsRhTi on the (010) and (001) surfaces, respectively. The corresponding 2D profiles of density of state (DOS) at fixed energies of -30 and 0 meV are also plotted in Fig. 3c, d. In Fig. 3a and Fig. 3c, one finds SSs spread throughout the inner region enclosed by the projection of $m-\mathrm{NL} \beta$ of Fig. 2d. Since the s-NLs are normal to surface (010) (see in Fig. 2d), no SS is found at (010) surface for the s-NLs. In contrast, on the (001) surface the projection of $s$-NLs form two ellipses on $\Gamma$ $X$ and SSs link the two ellipses across the boundary of BZ (see in Fig. 3d). It is seen from Fig. $3 d$ that the fermi surface also cuts some trivial bands, producing extra carrier pockets above and below the $k_{y}=0$ plane as can be seen in Fig. $3 \mathrm{~d}$.

In the above discussions, we have not included the spin-orbit coupling (SOC). The inclusion of SOC induces small gaps on the $\mathrm{NLs}$ at the scale of several meV and thus its effect can be ignored at room temperature. For compounds containing heavier elements below, such as SilrTa, the SOC gap is not small that the NLSMs eventually are turned into topological insulators. We also checked the effect of electron interactions by adopting the hybrid density functional approximation (HSE06) ${ }^{58}$ and find the HSE06 result reproduces GGA result very well (see in the Fig. 2b). One finds that HSEO6 calculation reproduces GGA band structure and makes the fermi surface even clearer by pushing down the valence band at $\Gamma$ and $U$.

Diverse node lines structures in MgSrSi-type crystals

The MgSrSi-type crystals is a large family of binary and ternary crystals which contains more than 660 members. As expected that 
(a)

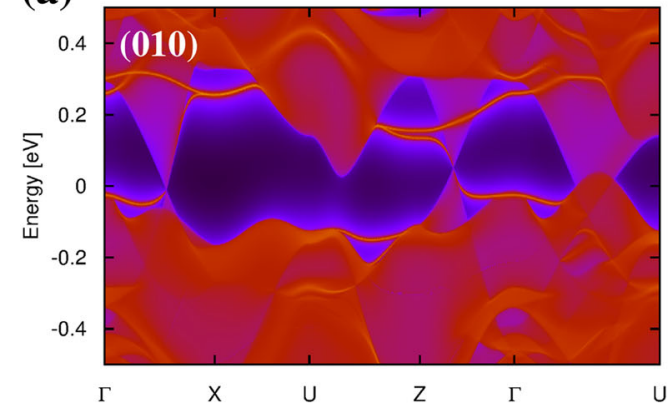

(c)

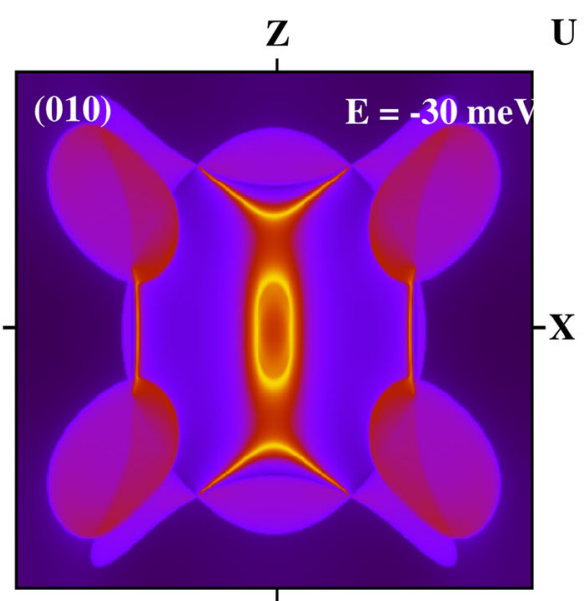

(b)

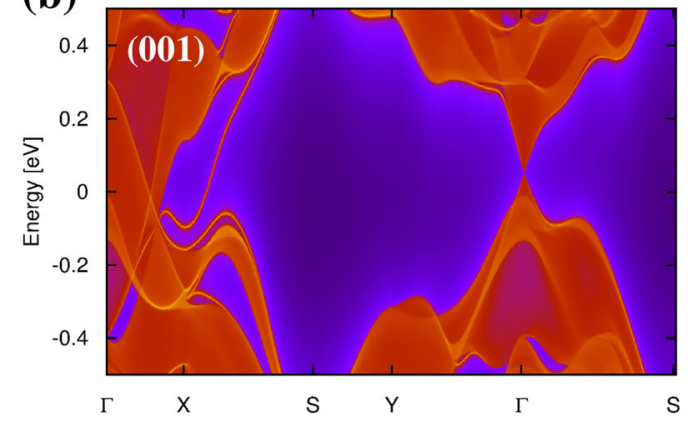

(d)

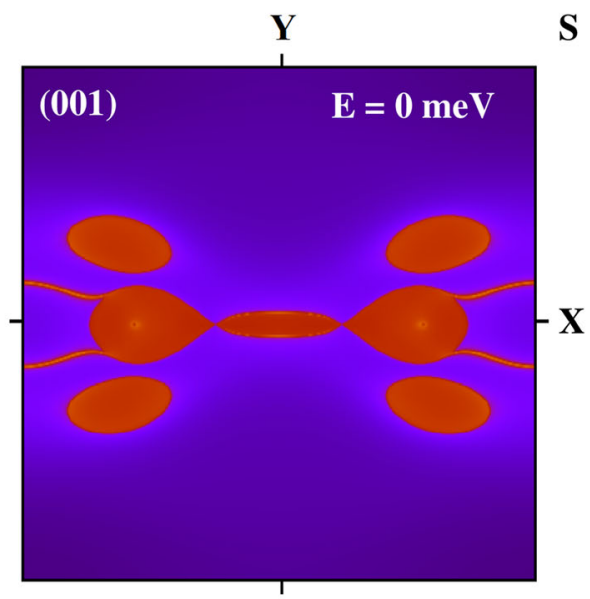

Fig. 3 Surface electronic structure of AsRhTi. a, b show the surface band structures on the (010) and (001) surfaces, respectively. c, $\mathbf{d}$ Plot the 2D surface DOS profiles corresponding to $\mathbf{a}$ and $\mathbf{b}$. The energies of $\mathbf{c}$ and $\mathbf{d}$ are set as -30 and $0 \mathrm{meV}$, respectively

\begin{tabular}{|c|c|}
\hline Catalog & Node-line semimetals \\
\hline $\mathrm{sRhTi}{ }^{46,47 a}$ & AsRhTi, AsCoHf, PCoHf, PCoTi, PCoZr, PRhZr \\
\hline $\mathrm{eV}^{48}$ & PFeV, PFeNb, PFeTa, AsFeNb, AsFeTa, PRuNb, PRuTa \\
\hline $\mathrm{NiV}^{49,50}$ & PNiV, PNiNb, PNiTa, AsNiV, AsNiNb, AsNiTa \\
\hline $\mathrm{PtSc}^{51}$ & PNiSc, PPtSc, PPdEr, AsPdLn ${ }^{b}(L n=L a, G d, H o, T b, D y, T m)$ \\
\hline $\mathrm{SiCoV}^{52,53}$ & $\begin{array}{l}\text { SiCoV, SiCoNb, SiCoTa, SiRhNb, SiRhTa, SilrNb, SilrTa, } \\
\text { GeCoV, GeCoNb, GeCoTa, GeRhNb, GeRhTa, GelrTa }\end{array}$ \\
\hline $\mathrm{SiNiTi}{ }^{54,55}$ & $\begin{array}{l}\text { SiNiTi, SiNiZr, SiNiHf, SiPdTi, SiPdZr, SiPdHf, SiPtTi, SiPtZr, } \\
\text { SiPtHf, GeNiZr, GeNiHf, GePdTi, GePdZr, GePtZr, GePtHf }\end{array}$ \\
\hline other $^{56 c}$ & $\begin{array}{l}\mathrm{GeBa}_{2}, \mathrm{SiBa}_{2}, \mathrm{PbBa}_{2}, \mathrm{PbCa}_{2}, \mathrm{SnBa}_{2}, \mathrm{SiMgBa}, \mathrm{SiMgCa} \\
\text { SiMgSr, SiCaBa, GeSrMg, SnCaMg, SrMgSn, CaBiLi }\end{array}$ \\
\hline \multicolumn{2}{|c|}{ 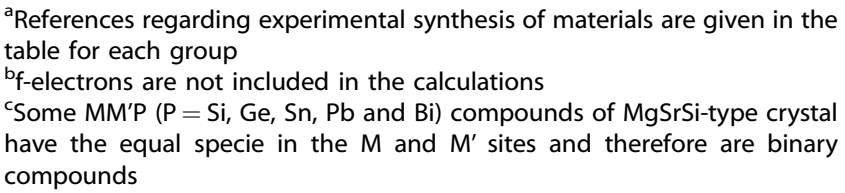 } \\
\hline
\end{tabular}

isostructure crystals may have similar electronic structure, we thus screened all 660 compounds to discover new NLSMs. More than 70 NLSMs are readily found and listed in Table 1, where the NLSMs are divided into several groups based on their chemical compositions. A variety of NLs structures were discovered. The NL structures for each group is similar. In Fig. 4 we plotted 5 representative NL structures and their corresponding fermi surfaces. From Fig. 4a, one sees PPtSc has a single NL loop lying in the $k_{y}=0$ plane and its fermi surface takes a distorted torus-like shape (see in Fig. 4a). For SiNiZr, the NL extends across the boundary of $B Z$ and one can see from Fig. $4 b$ that some portions of the NL outside the BZ is folded back. The single NL of SiCoV shown in Fig. 4c, contrarily, lies on the $k_{z}=0$ plane unlike those of $\mathrm{PPtSC}$ and SiNiZr in the $k_{y}=0$ plane, indicating its protecting symmetry being $\tilde{m}_{z}$. Both fermi surfaces of PPtSc and SiCoV take the simple torus-like shapes and show very clean fermi surfaces, a promising property for the experimental detection of their nontrivial electronic structures.

Interestingly, even more exotic NL geometries are found in AsFeNb and PNiNb. There exist multiple $m$-NLs in the $k_{x}=0, k_{y}=$ 0 , and $k_{z}=0$ planes, together with $s$-NLs sticking to the $m$-NLs (see in Fig. 4d, e). For AsFeNb, its NLs form a novel cage-like structure. However, its fermi surface is dirty which is messed up by some trivial bands. For PNiNb, one finds an isolated $m$-NL lies in the invariant plane $k_{y}=0$, and off the plane NLs protected by $\tilde{m}_{x}$ and $\tilde{m}_{z}$ are found touching near $Y$ point. Around the planes of $k_{x}= \pm k_{z}$ there exist eight segments of $s$-NLs sticking to the $m$-NLs of $k_{x}=0$ plane. Luckily the fermi surface of $\mathrm{PNiNb}$, shown in Fig. $4 \mathrm{j}$, is very clean and quite similar to the NLs structure of Fig. 4e. Therefore $\mathrm{PNiNb}$ can be a promising compound for exploring new NLSMs with exotic multi-loop NL structure.

From the summarized result in Table 1, we find that the electron counting is a useful indicator for the search of NLSMs. The number of electrons, being totally 32 electrons/unit cell according to the counting scheme of Landrum et al., ${ }^{54}$ are all the same for the $\mathrm{AsRhTi}^{G}, \mathrm{PFeV}^{G}, \mathrm{PNiV}^{G}, \mathrm{SiCoV}^{G}$, and $\mathrm{SiNiTi}^{G}$ groups, while the $\mathrm{PPtSc}^{G}$ has 40 electrons/unit cell. In other words, all the NLSMs listed in Tab. 1 has an count of 8 electrons per formula unit. According to a simple 8-N rules of Zintl, one may expect that a $\mathrm{NiSiTi}$ should be an insulator or semiconductor. ${ }^{59}$ It is the strong bonding of transition metal atoms of SiNiTi leads to the semiemtal 
(a)

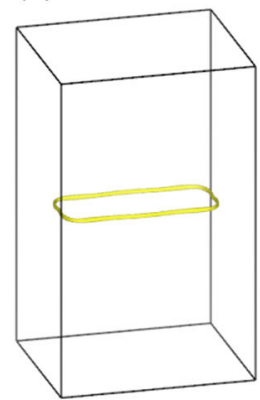

PPtSc

(f)

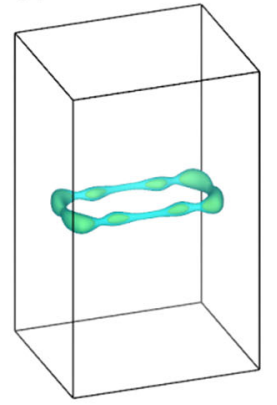

(b)

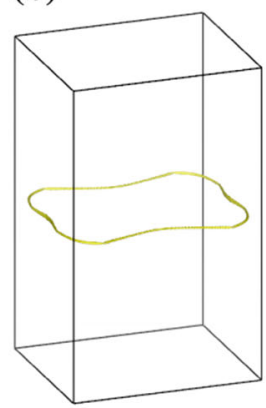

SiNiZr

(g)

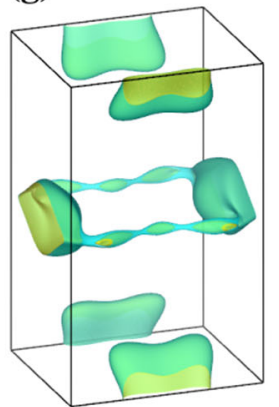

(c)

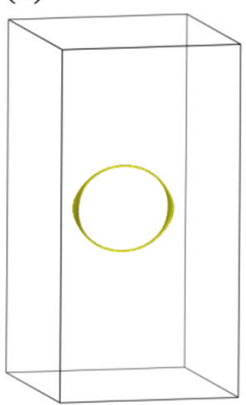

SiCoV

(h)

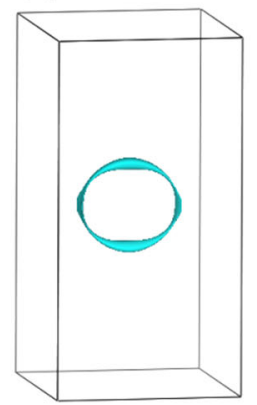

(d)

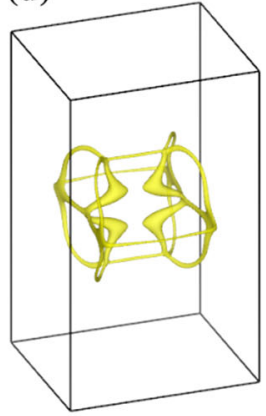

AsFeNb (e)

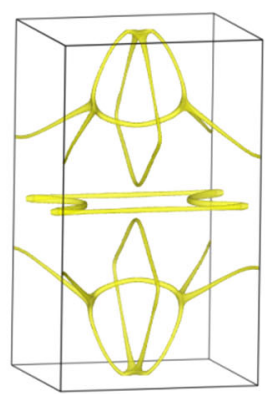

PNiNb (i)

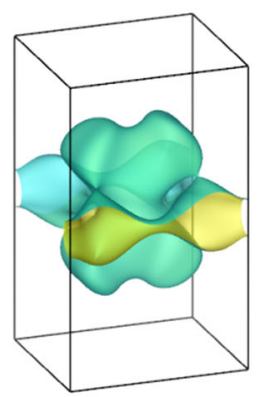

(j)

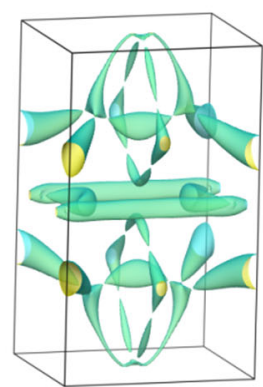

Fig. 4 A variety of structures of NLs in MgSrSi-type ternary crystals. a-e Plot the band crossings of PPtSc, SiNiZr, SiCoV, AsFeNb, and PNiNb. f-j The corresponding fermi surfaces of PPtSc, SiNiZr, SiCoV, AsFeNb, and PNiNb

state rather than an insulating one. ${ }^{54}$ Here our high-throughput calculations and screening of NLSMs in MgSrSi series teach us that semimetals with filled octet orbitals could be good candidate of topological SMs.

It is worthy to note that many MgSrSi-type crystals, though containing magnetic atoms, are actually non-magnetic. For example, the ternary $\mathrm{MMX}\left(\mathrm{M}=\right.$ transition metal, $\mathrm{M}^{\prime}=$ late transition metal, $X=$ main group element) compounds exhibit paramagnetic behaviors for $\mathrm{M}=\mathrm{Sc}, \mathrm{Ti}$ and $\mathrm{V} .{ }^{54}$ Previously, TiCoP, $\mathrm{ZrCoP}$, and $\mathrm{VCoSi}$ have already been characterized to be paramagnetic metallic conductors. The trends of nonmagnetic ground state of MgSrSi-type crystal with open-shell magnetic atoms is attributed to the strong bonding of $\mathrm{M}-\mathrm{M}^{\prime}$ by Goodenough that the $\mathrm{M}-\mathrm{M}^{\prime}$ bonding energy of the MgSrSi-type ternary may dominate over the intra-atomic Coulomb interaction which favors magnetic state in the free-atom limit. ${ }^{60}$

In conclusion, we screen the MgSrSi-type crystals and more than 70 compounds are found to host node line band crossing in their band structures. Due to the coexistence of reflection symmetry and inversion symmetry in the space group, AsRhTi is found to take a novel multi-loop NL structure, in which a $s-\mathrm{NL}$ protected by PT symmetry touches robustly with a $m$-NL protected by mirror symmetry at some "nexus point", and in the meanwhile a second $m$-NL crosses with the $s$-NL to form a Hopf-link. A essential three-band $k p$ model is provided to give an effective description of the low energy electrons on the Fermi surface. Topological surface states exhibiting the nontrivial NL structure is also demonstrated. Even more exotic multi-loop NL structures are uncovered in AsFeNb and PNiNb sub-groups. All the found NLSMs are consistent with 8-N valence rules of Zintl, indicating the valence rules should be an useful indicator for the searching of topological SMs.

\section{METHODS}

The first principles calculations were performed by the Vienna ab initio simulation package (VASP) ${ }^{61}$ and the projected augmented-wave (PAW) potential is adopted. ${ }^{62,63}$ The exchange-correlation functional introduced by Perdew, Burke, and Ernzerhof (PBE) ${ }^{64}$ within generalized gradient approximation (GGA) is applied in the calculations. The energy cutoff for the plane-wave basis is set as $520 \mathrm{eV}$ and the forces are relaxed less than $0.01 \mathrm{eV} / \AA$. . The positions of atoms are allowed to relax while the lattice constants of the unit cells are fixed to the experimental values documented in the Inorganic Crystal Structure Database (ICSD). The band-crossings are calculated from tight-binding models which are constructed by using the Maximally Localized Wannier Functions (MLWF) method coded in WANNIER90. ${ }^{65}$ To give a more accurate description of electron interaction, hybrid density functional approximation is further adopted. ${ }^{58}$

\section{DATA AVAILABILITY}

The data that support the findings of this study are available from the corresponding authors upon reasonable request.

\section{ACKNOWLEDGEMENTS}

The work is supported by National Natural Science Foundation of China (NSFC) (Grants No. 11574215, No. 11575116, No. 11274359, and No. 11422428). H. M. W is also supported by the National 973 program of China (Grants No. 2018 YFA0305700 and No. 2013CB921700), and the "Strategic Priority Research Program (B)" of the Chinese Academy of Sciences (Grant No. XDB07020100). The calculations in this work were performed on the supercomputers of Shanghai supercomputer Center and of the high performance computing center of Nanjing University.

\section{AUTHOR CONTRIBUTIONS}

Q.-F.L., R.Y., and H.M.W. conceived the study. J.L. and L.Y. wrote the necessary code, J. L., L.Y., and Q.-F.L. carried out the calculations. Q.-F.L. analyzed the data. All four authors contributed to writing the manuscript. J.L. and L.Y. contributed equally to this work. 


\section{ADDITIONAL INFORMATION}

Competing interests: The authors declare no competing interests.

Publisher's note: Springer Nature remains neutral with regard to jurisdictional claims in published maps and institutional affiliations.

\section{REFERENCES}

1. Weng, H., Dai, X. \& Fang, Z. Topological semimetals predicted from first-principles calculations. J. Phys. Condens. Matter 28, 303001 (2016).

2. Fang, C., Weng, H., Dai, X. \& Fang, Z. Topological nodal line semimetals. Chin. Phys. B 25, 9-18 (2016).

3. Wan, X., Turner, A. M., Vishwanath, A. \& Savrasov, S. Y. Topological semimetal and fermi-arc surface states in the electronic structure of pyrochlore iridates. Phys. Rev. B 83, 205101 (2011).

4. Burkov, A. A. \& Balents, L. Weyl semimetal in a topological insulator multilayer. Phys. Rev. Lett. 107, 127205 (2011).

5. Xu, G., Weng, H., Wang, Z., Dai, X. \& Fang, Z. Chern semimetal and the quantized anomalous hall effect in hgcr $_{2} \mathrm{Se}_{4}$. Phys. Rev. Lett. 107, 186806 (2011).

6. Weng, H., Fang, C., Fang, Z., Bernevig, B. A. \& Dai, X. Weyl semimetal phase in noncentrosymmetric transition-metal monophosphides. Phys. Rev. X 5, 011029 (2015).

7. Lv, B. Q. et al. Experimental discovery of weyl semimetal taas. Phys. Rev. X 5, 031013 (2015).

8. $\mathrm{Xu}, \mathrm{S}$.-Y. et al. Topological matter: discovery of a weyl fermion semimetal and topological fermi arcs. Science 349, 613-617 (2015).

9. Murakami, S. Gap closing and universal phase diagrams in topological insulators. Phys. E 43, 748-754 (2011).

10. Wang, Z. et al. Dirac semimetal and topological phase transitions in $A_{3} \mathrm{bi}(\mathrm{a}=\mathrm{Na}$, k, rb). Phys. Rev. B 85, 195320 (2012).

11. Wang, Z., Weng, H., Wu, Q., Dai, X. \& Fang, Z. Three-dimensional dirac semimetal and quantum transport in $\mathrm{Cd}_{3} \mathrm{as}_{2}$. Phys. Rev. B 88, 125427 (2013).

12. Burkov, A. A., Hook, M. D. \& Balents, L. Topological nodal semimetals. Phys. Rev. $B$ 84, 235126 (2011).

13. Weng, $\mathrm{H}$. et al. Topological node-line semimetal in three-dimensional graphene networks. Phys. Rev. B 92, 045108 (2015).

14. Kim, Y., Wieder, B. J., Kane, C. L. \& Rappe, A. M. Dirac line nodes in inversionsymmetric crystals. Phys. Rev. Lett. 115, 036806 (2015).

15. Yu, R., Weng, H., Fang, Z., Dai, X. \& Hu, X. Topological node-line semimetal and dirac semimetal state in antiperovskite $\mathrm{Cu}_{3} \mathrm{PdN}$. Phys. Rev. Lett. 115, 036807 (2015).

16. Xie, L. S. et al. A new form of $\mathrm{Ca}_{3} \mathrm{P}_{2}$ with a ring of dirac nodes. APL Mat. 3, 083602 (2015).

17. Bian, G. et al. Drumhead surface states and topological nodal-line fermions in tltase 2 . Phys. Rev. B 93, 121113 (2016).

18. Bian, G. et al. Topological nodal-line fermions in spin-orbit metal pbtase2. Nat. Commun. 7, 10556 (2016).

19. Heikkila, T. T. \& Volovik, G. E. Flat bands as a route to high-temperature superconductivity in graphite. arXiv: 1504.05824 (2015).

20. Fang, C., Chen, Y., Kee, H.-Y. \& Fu, L. Topological nodal line semimetals with and without spin-orbital coupling. Phys. Rev. B 92, 081201 (2015).

21. Phillips, M. \& Aji, V. Tunable line node semimetals. Phys. Rev. B 90, 115111 (2014).

22. Weber, S. F., Chen, R., Yan, Q. \& Neaton, J. B. Prediction of tirhas as a dirac nodal line semimetal via first-principles calculations. Phys. Rev. B 96, 235145 (2017).

23. Huang, H., Liu, J., Vanderbilt, D. \& Duan, W. Topological nodal-line semimetals in alkaline-earth stannides, germanides, and silicides. Phys. Rev. B 93, 201114 (2016).

24. Huang, H., Jin, K.-H. \& Liu, F. Topological nodal-line semimetal in nonsymmorphic Crece -phase $\mathrm{Ag}_{2}$ S. Phys. Rev. B 96, 115106 (2017).

25. Li, R. et al. Dirac node lines in pure alkali earth metals. Phys. Rev. Lett. 117, 096401 (2016).

26. Neupane, M. et al. Observation of topological nodal fermion semimetal phase in zrsis. Phys. Rev. B 93, 201104 (2016).

27. $\mathrm{Hu}$, J. et al. Evidence of topological nodal-line fermions in zrsise and zrsite. Phys. Rev. Lett. 117, 016602 (2016).

28. Bzdušek, T., Wu, Q., Rüegg, A., Sigrist, M. \& Soluyanov, A. A. Nodal-chain metals. Nature 538, 75 (2016).

29. Yu, R., Wu, Q., Fang, Z. \& Weng, H. From nodal chain semimetal to weyl semimetal in hfc. Phys. Rev. Lett. 119, 036401 (2017).

30. Xu, Q., Yu, R., Fang, Z., Dai, X. \& Weng, H. Topological nodal line semimetals in the $\mathrm{cap}_{3}$ family of materials. Phys. Rev. B 95, 045136 (2017).

31. Quan, Y., Yin, Z. P. \& Pickett, W. E. Single nodal loop of accidental degeneracies in minimal symmetry: triclinic caas $_{3}$. Phys. Rev. Lett. 118, 176402 (2017).
32. Yan, Z. et al. Nodal-link semimetals. Phys. Rev. B 96, 041103 (2017).

33. Bi, R., Yan, Z., Lu, L. \& Wang, Z. Nodal-knot semimetals. Phys. Rev. B 96, 201305 (2017).

34. Chen, W., Lu, H.-Z. \& Hou, J.-M. Topological semimetals with a double-helix nodal link. Phys. Rev. B 96, 041102 (2017).

35. Chang, G. et al. Topological hopf and chain link semimetal states and their application to $\mathrm{CO}_{2} \mathrm{MnGa}$. Phys. Rev. Lett. 119, 156401 (2017).

36. Zhou, Y., Xiong, F., Wan, X. \& An, J. Hopf-link topological nodal-loop semimetals. Phys. Rev. B 97, 155140 (2018).

37. Huang, H., Jiang, W., Jin, K.-H. \& Liu, F. Tunable topological semimetal states with ultraflat nodal rings in strained yn. Phys. Rev. B 98, 045131 (2018).

38. Wang, J.-T., Nie, S., Weng, H., Kawazoe, Y. \& Chen, C. Topological nodal-net semimetal in a graphene network structure. Phys. Rev. Lett. 120, 026402 (2018).

39. Wu, Q., Zhang, S., Song, H.-F., Troyer, M. \& Soluyanov, A. A. Wanniertools: an open-source software package for novel topological materials. Comput. Phys. Commun. 224, 405-416 (2018).

40. Wang, S.-S., Liu, Y., Yu, Z.-M., Sheng, X.-L. \& Yang, S. A. Hourglass dirac chain metal in rhenium dioxide. Nat. Commun. 8, 1844 (2017).

41. Zhong, C. et al. Three-dimensional pentagon carbon with a genesis of emergent fermions. Nat. Commun. 8, 15641 (2017).

42. Liang, Q.-F., Zhou, J., Yu, R., Wang, Z. \& Weng, H. Node-surface and node-line fermions from nonsymmorphic lattice symmetries. Phys. Rev. B 93, 085427 (2016).

43. Zhang, $X$. et al. Nodal loop and nodal surface states in the $t_{3} A l$ family of materials. Phys. Rev. B 97, 235150 (2018).

44. Heikkila, T. T. \& Volovik, G. E. Nexus and dirac lines in topological materials. New J. Phys. 17, 093019 (2015).

45. Yan, Q. et al. Experimental discovery of nodal chains. Nat. Phys. 14, 461-464 (2018).

46. Watts, G. Rh Rhodium 107-114 (Springer, 1991).

47. Suzuki, N., Asahi, R., Kishida, Y., Masuoka, Y. \& Sugiyama, J. Measurement and ab initio calculation of the structural parameters and physical properties of $3 \mathrm{~d}$ transition intermetallics timp $(\mathrm{m}=\mathrm{cr}, \mathrm{mn}, \mathrm{fe}, \mathrm{co}$, or ni). Mater. Res. Express 4, 046505 (2017).

48. Roy-Montreuil, M. et al. Nouveaux composes ternaires $\mathrm{mm}^{\prime} \mathrm{p}$ et $\mathrm{mm}$ 'as interactions metalliques et structures. Mater. Res. Bull. 7, 813-826 (1972).

49. Pies, W. \& Weiss, A. c1341, VII.1.2.1 Binary and ternary phosphides, 45-60 (Springer, Berlin Heidelberg, 1979).

50. Johnson, V. Ternary transition metal germanides and arsenides. Mater. Res. Bull. 8, 1067-1072 (1973).

51. Imre, A. \& Mewis, A. Scptp und laptp-zwei phosphide mit, inverser tinisi-struktur/ scptp and laptp-two phosphides with inverse tinisi-type structure. Z. für Naturforsch. B 62, 1153-1156 (2007).

52. Dinges, T., Eul, M. \& Poettgen, R. Tarhge with tinisi-type structure. Z. für Naturforsch. B 65, 95-98 (2010).

53. Mishra, R., Poettgen, R. \& Kotzyba, G. New metal-rich compounds nblrsi, nblrge, and talrsi-synthesis, structure, and magnetic properties. Z. für Naturforsch. B 56, 463-468 (2001).

54. Landrum, G. A., Hoffmann, R., Evers, J. \& Boysen, H. The tinisi family of compounds: structure and bonding. Inorg. Chem. 37, 5754-5763 (1998).

55. Zhao, J. \& Parth, E. Ternary equiatomic hafnium-transition metal suicides. J. Less Common Met. 163, L7-L12 (1990).

56. Gil-Santos, A., Szakacs, G., Moelans, N., Hort, N. \& Van der Biest, O. Microstructure and mechanical characterization of cast mg-ca-si alloys. J. Alloy Compd 694, 767-776 (2017).

57. Rui, W. B., Zhao, Y. X. \& Schnyder, A. P. Topological transport in Dirac nodal-line semimetals. arXiv: 1703.05958 (2017)

58. Heyd, J., Scuseria, G. E. \& Ernzerhof, M. Erratum: hybrid functionals based on a screened coulomb potential. J. Chem. Phys. 124, 219906 (2006).

59. Zintl, E. Intermetallische verbindungen. Angew. Chem. 52, 1-6 (1939).

60. Goodenough, J. Interpretation of structure and magnetism in transition-metal pnictides m2x and (m1- xmjä x) 2x. J. Solid State Chem. 7, 428-447 (1973).

61. Kresse, G. \& Furthmüller, J. Efficient iterative schemes for ab initio total-energy calculations using a plane-wave basis set. Phys. Rev. B 54, 11169-11186 (1996).

62. Blöchl, P. E. Projector augmented-wave method. Phys. Rev. B 50, 17953-17979 (1994).

63. Kresse, G. \& Joubert, D. From ultrasoft pseudopotentials to the projector augmented-wave method. Phys. Rev. B 59, 1758-1775 (1999).

64. Perdew, J. P., Burke, K. \& Ernzerhof, M. Generalized gradient approximation made simple. Phys. Rev. Lett. 77, 3865-3868 (1996).

65. Mostofi, A. A. et al. wannier90: a tool for obtaining maximally-localised wannier functions. Comput. Phys. Commun. 178, 685 (2008). 
Open Access This article is licensed under a Creative Commons Attribution 4.0 International License, which permits use, sharing, adaptation, distribution and reproduction in any medium or format, as long as you give appropriate credit to the original author(s) and the source, provide a link to the Creative Commons license, and indicate if changes were made. The images or other third party material in this article are included in the article's Creative Commons license, unless indicated otherwise in a credit line to the material. If material is not included in the article's Creative Commons license and your intended use is not permitted by statutory regulation or exceeds the permitted use, you will need to obtain permission directly from the copyright holder. To view a copy of this license, visit http://creativecommons. org/licenses/by/4.0/.

(c) The Author(s) 2019 